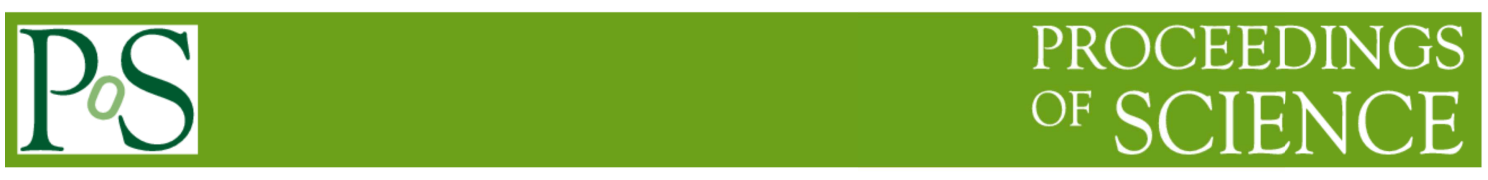

\title{
SALT observations of X-ray transients
}

\section{David A.H. Buckley ${ }^{1}$}

South African Astronomical Observatory

PO Box 9, Observatory 7935, Cape Town, South Africa

E-mail: dibnoblsaao.ac.za

\section{Philip A. Charles}

Department of Physics and Astronomy, Faculty of Physical Sciences and Engineering University of Southampton, Southampton SO17 1BJ, UK

E-mail: pac2sotonegmail.com

\section{Andry Rajoelimanana}

Department of Physics, University of the Free State

205 Nelson Mandela Drive, Park West, Bloemfontein, 9301, Republic of South Africa

E-mail: andrylsaao.ac.za

\section{Lee J. Townsend}

Department of Astronomy, University of Cape Town Private Bag X3, Rondebosch, 7701, South Africa

E-mail: townsendeast.uct.ac.za

This paper presents some highlights of results on X-ray transients which have been obtained as part of the SALT Large Science Programme on transient followup. These include both High and Low Mass X-ray binaries with either accreting neutron stars or black holes, as well the white dwarf accreting Super Soft Sources.

5th Annual Conference on High Energy Astrophysics in Southern Africa 4-6 October, 2017

University of the Witwatersrand (Wits), South Africa

\footnotetext{
${ }^{1}$ Speaker
} 


\section{Introduction}

The study of astrophysical transients, over all wavelengths, time and distance scales in the Universe, has entered a new era with the establishment of a number of ground- and space-based facilities, used in both detection and follow-up programmes. Many of these target specific classes of transients, for example supernovae (e.g. ZTF and ASAS-SN) and Gamma-Ray Bursts (e.g. ROTSE, GROND and Watcher). Other successful optical transient detection systems, designed for a wider range of object classes, include OGLE, CRTS and MASTER. Other survey facilities are discovering transients as a byproduct of their main science goals (e.g. PanSTARRs, Gaia and OGLE), as will also happen with future facilities (e.g., LSST, MeerKAT and SKA). Together with existing and upcoming X- and $\gamma$-ray and multi-messenger (gravitational wave and neutrino) observatories (e.g. Swift, Fermi, INTEGRAL, MAXI, ASTROSAT, NuSTAR, NICER, eROSITA, aLIGO/Virgo, IceCube), the opportunity to study the transient Universe is unprecedented. The volume of future alerts will grow immensely and will require machine learning techniques in order to select the most compelling objects for more intensive study. Lessons learned from the current SALT transient programme will help to inform the future automated selection processes to be used for these large-scale transient surveys, like LSST.

Here we discuss one aspect of the on-going SALT transient follow-up programme, specifically the class of X-ray transients (XRTs), which include 1.) low mass X-ray binaries (LMXBs), 2.) high mass X-ray binaries (HMXBs) and 3.) supersoft X-ray sources (SSSs). The first two contain compact accreting objects which are either neutron stars (NS) or black holes (BH), which go through outburst cycles producing hot accretion disks and jets. SSSs contain white dwarfs (WD) accreting at a high enough rate to sustain stable thermonuclear burning on their surfaces. This can drastically increase the temperature of the white dwarf photosphere (up to $\sim 10^{6} \mathrm{~K}$ ), resulting in a thermal spectrum emitting predominantly at extreme ultraviolet and soft X-ray energies. Some cataclysmic variable also undergo transient X-ray emission, due to accretion disk instabilities (i.e. dwarf novae) or nova outbursts (i.e. classical and recurrent novae), but these will not be discussed here.

\section{Observing Transients with SALT}

SALT is a fully queue-scheduled telescope, which makes it ideal to undertake target of opportunity (ToO) observations, as is often required for transient followup, more easily than traditionally scheduled telescopes. The main considerations in conducting SALT followup observations is target availability and the timescale over which such observations can be attempted. Because of SALT's design, namely the fixed altitude angle of the telescope structure, the instantaneous viewing window for SALT is only $\sim 12 \%$ of the visible sky available to SALT at any given time (defined as that part of the celestial hemisphere which above $20^{\circ}$ elevation). Observations therefore have to be carefully planned so objects of interest are accessible to SALT. For prompt followup observations (e.g. < few $1000 \mathrm{~s}$ from an alert), like gamma ray bursts, this can be challenging. Fortunately many transients, including X-ray transients (XRTs, consisting of LMXBs, HMXBs and SSSs), evolve over periods of weeks to months, which lend themselves to regular monitoring on a cadence of a days or so. The SALT transient programme has been able to undertake such regular follow-up observations. 
The instruments on SALT are well suited to a variety of different follow-up observations of transients, particularly spectroscopy for the characterization of the object and time resolved studies. The four science instruments on SALT [1] being utilized for X-ray transient studies comprise of the following:

1.RSS, the Robert Stobie Spectrograph, a versatile prime focus instrument supporting:

- low to medium resolution long-slit spectroscopy $(\mathrm{R} \sim 300-6000)$

- spectropolarimetry and imaging polarimetry (linear and circular)

- high time resolution (0.1s) spectroscopy and photometry

2.SALTICAM, a broad-band UV-visible imaging camera, which supports:

- moderately high time resolution (1 s) frame transfer CCD photometry over a $\sim 4$ x 8 arcmin field

- high time resolution $(0.1 \mathrm{~s})$ frame transfer CCD photometry over a $20 \operatorname{arcsec} \times 8$ arcmin field

3.BVIT, the Berkeley Visible Image Tube photon counting camera (a visitor instrument):

- photon counting camera using a microchannel plate and S20 photocathode time tagging events to $50 \mathrm{~ns}$

\section{Rationale for observing X-ray transients}

The transient X-ray sky is providing an important window into the properties of both LMXBs and HMXBs, with $~ 75 \%$ of the former recognised as BH candidates [2], and the latter linked to the newly-discovered NS class of ULX pulsars [3]. They hold the prospect to provide key information on orbital solutions and system masses, as well as constraining massive stellar evolutionary processes. Accreting millisecond X-ray pulsars can constrain the equation of state of super-dense NS matter and are the "missing link" in the evolutionary sequence from X-ray binaries (XRBs) to millisecond radio pulsars, and are only detected as XRTs.
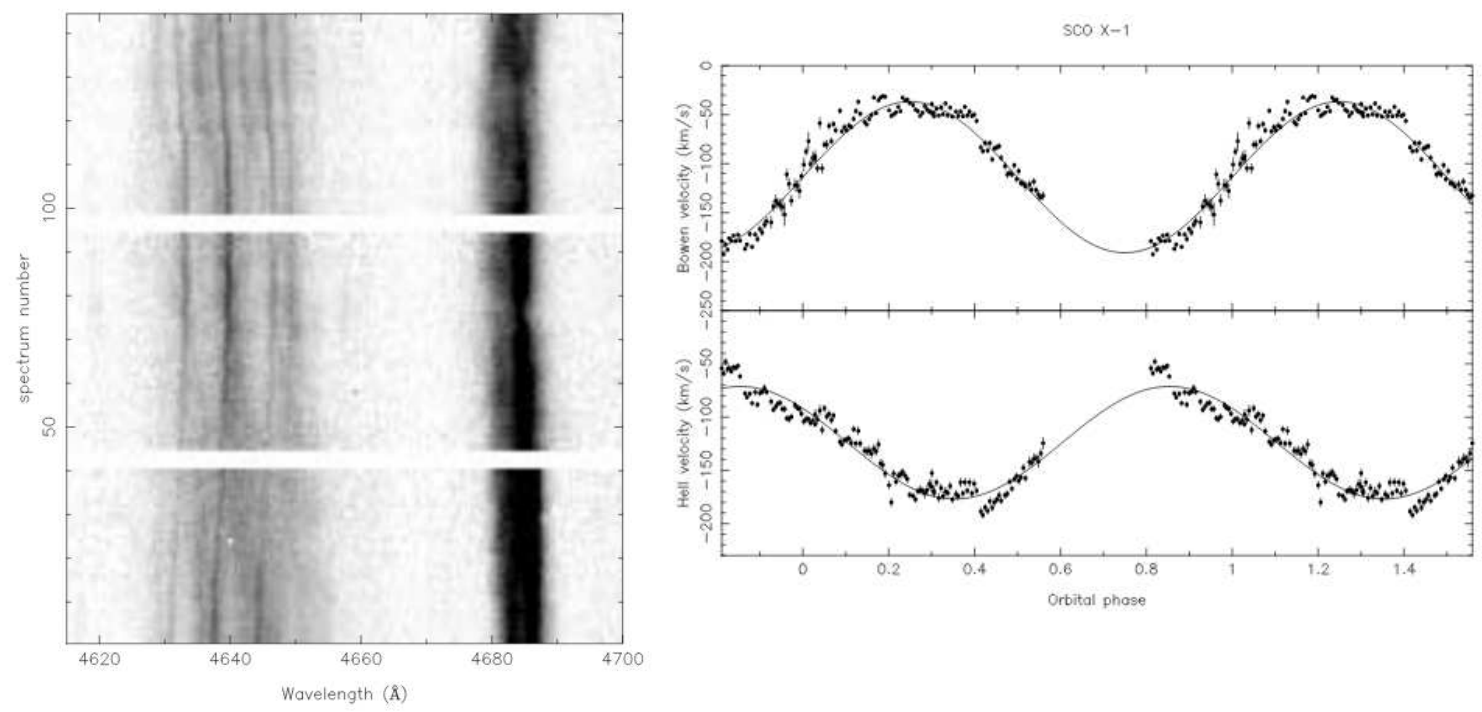

Figure 1: Trailed spectra (left) and radial velocity curves (right) of the Bowen (upper) and HeII (lower) emission from Sco X-1 ${ }^{[4]}$. The former emanate from the irradiated secondary star while the latter are from the accretion disk. 
A new avenue for obtaining direct dynamical information was opened via phase-resolved, high resolution spectroscopy of the proto-typical LMXB, Sco X-1 [4]. Narrow emission-line components can be seen (Fig. 1; left panel) arising from the irradiated face of the donor, thereby establishing the absolute orbital ephemeris and the first radial velocity curve for the Sco X-1 secondary (Fig. 1; right panel) [4]. These narrow features were most obvious in the Bowen

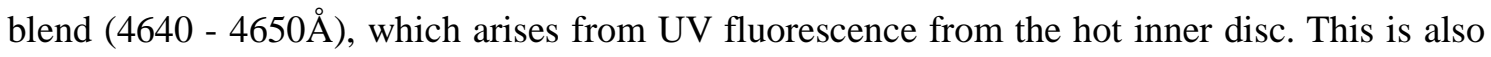
seen in other LMXBs and is now known to be a common phenomenon in all such systems [4]. Although the importance of irradiation of the donor star in X-ray binaries is well established, this technique has only recently been exploited, because of the need for high spectral resolution on faint targets.

When in quiescence, LMXB XRTs offer the possibility of detailed dynamical studies to establish key binary properties, the most important of which is the compact object mass, which has only been done for 20 systems to date [5,6]. The SALT XRT program, together with complementary programmes by our collaborators on other telescopes, e.g. on the 10.4-m Gran Telescopio Canarias (GTC), has been invaluable for studying many of the XRTs that occurred since 2016 (see Table 1, which shows the wide range of new XRTs that have been observed during this programme), and demonstrates the importance of rapid follow-up of new XRTs. As anticipated, most of the alerts of XRTs have come from X-ray satellite notifications (e.g. Swift, MAXI), some for which we have obtained valuable multi-wavelength follow-up observations with other X-ray facitilies (e.g. ASTROSAT and NuSTAR).

Be X-ray binaries (transient HMXBs), almost all of which are neutron star (NS) systems (many found as X-ray pulsars), have been observed during and after X-ray outbursts. These observations assist us in understanding the mass transfer processes, which are driven by the $\mathrm{Be}$ star circumstellar disk. The most spectacular so far was the mid-2016 giant Type II superEddington outburst of SMC X-3, which peaked at $\sim 10^{39} \mathrm{erg} \mathrm{s}^{-1}$, making this one of only two known ULX X-ray pulsars in the Local Group [8]. Although there are predicted to be a significant population of Be-white dwarf systems, only a handful have been discovered to date e.g. the transient super-soft Source in the SMC [9].

\section{Results}

The X-ray transients which we have observed to date are presented in Table 1. In the following subsections we present a sample of results relating to: 1.) a SSS (ASASSN-160h), 2.) two LMXBs, one a NS system (MAXI J1957+032) and the other a BH system (Swift J1357.20933) and 3.) a HMXB (the Be /X-ray binary SMC X-3).

\begin{tabular}{|l|l|l|}
\hline \multicolumn{1}{|c|}{ Object } & \multicolumn{1}{|c|}{ Transient event } & \multicolumn{1}{c|}{ Status/Publications } \\
\hline SMC X-3 & $\begin{array}{l}\text { 2016.7.3 type II outburst (ULX-level) } \\
\text { in HMXB }\end{array}$ & Townsend et al 2017 MN 471, 3878 \\
\hline MAXI J1957+032 & $\begin{array}{l}2016.10 .1 \text { V 18 - >21 likely new } \\
\text { AMXP }\end{array}$ & Mata Sanchez et al 2017 MN 468, 564 \\
\hline ASASSN-16oh & $\begin{array}{l}2016.12 .14 \text { V 21-16 new SSS in } \\
\text { SMC }\end{array}$ & $\begin{array}{l}\text { Maccarone et al 2018 [15] (in press); } \\
\text { Rajoelimanana et al 2018, in prep. }\end{array}$ \\
\hline $\begin{array}{l}\text { GRS1716-249 (N Oph } \\
\text { 93) }\end{array}$ & $\begin{array}{l}2017.3 .1 \text { Known BHXRB (+GTC + } \\
\text { VLT-Xshooter) }\end{array}$ & Analysis continuing (IAC lead) \\
\hline PSR J1023+0038 & 2017.6 .13 & Object too faint \\
\hline
\end{tabular}




\begin{tabular}{|l|l|l|}
\hline Swift J1753.5-0127 & 2017.4.20 final outbursts & Paice et al 2018, in prep. \\
\hline Swift J1357.2-0933 & 2017.4.21 $2^{\text {nd }}$ outburst (+NuSTAR) & Paice et al 2018, in prep. \\
\hline Aq1 X-1 & 2017.6.24 Known NS XRT & $\begin{array}{l}\text { Spectra and photometry under } \\
\text { investigation }\end{array}$ \\
\hline 4U1543-624 & $\begin{array}{l}\text { LMXB with 18 min period; 2017.8.19 } \\
\text { Swift x6 increase, opt faint }\end{array}$ & Too faint \\
\hline OGLE-17nif & $\begin{array}{l}\text { 2017.8.30 V 18.5 new SSS, also in } \\
\text { SMC, similar to ASASSN-16oh }\end{array}$ & $\begin{array}{l}\text { Analysis continuing. New season } \\
\text { observations needed. }\end{array}$ \\
\hline MAXI J1535-571 & $\begin{array}{l}\text { 2017.9.8 V>20 SALTICAM, } \\
\text { AstroSAT }\end{array}$ & Baglio et al. 2018 (accepted) \\
\hline GX339-4 & 2017.9.29 HRS, SALTICAM & ATel \#10820, 10864; paper in prep. \\
\hline
\end{tabular}

Table 1: X-ray transient observations undertaken to date as part of SALT transients programme.

\subsection{The Super Soft Source ASASSN-16oh}

This object, situated in the Small Magellanic cloud, was found by the ASAS-SN survey [10] and observed by SALT [11] to have a very blue spectrum, dominated by intense and relatively narrow HeII 4686 emission (see Figure 2). Peaking at around 15th magnitude, the spectrum is typical of a SSS (Super Soft Source), which are usually interpreted as superEddington accreting white dwarfs undergoing continuous thermonuclear burning on their surfaces. X-ray spectra taken with the Neil Gehrels Swift observatory immediately following discovery confirmed its soft X-ray nature, establishing it as one of a handful of SSS known in the Magellanic Clouds.

During the period that ASASSN-16oh was accessible to SALT, we obtained 25 spectra on 9 different nights, from 16-30 December 2016. They revealed small radial velocity changes in the strong HeII line. We also arranged for more intensive photometry from the Las Cumbres Observatory (LCO), with $\sim 20 \mathrm{~h}$ allocated from a DDT programme, to probe its short-term variability. Coordinated followup observations with ASTROSAT (the first of a SSS) were also conducted, resulting in a X-ray/UV spectrum of the object.

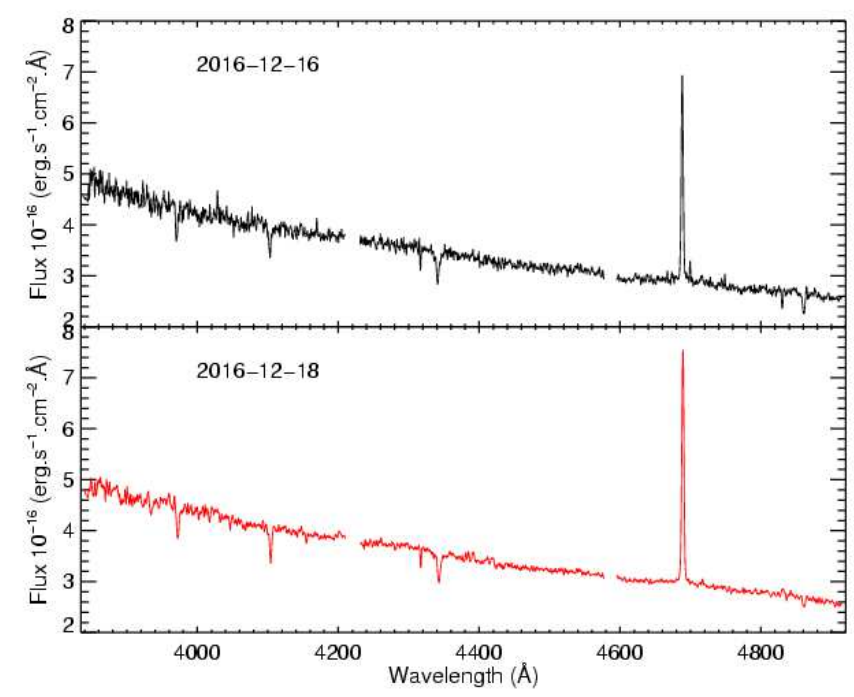

Figure 2: Blue SALT spectra (PG2300) of ASASSN-16oh taken during outburst. The emission line is HeII $4686 \AA$. 


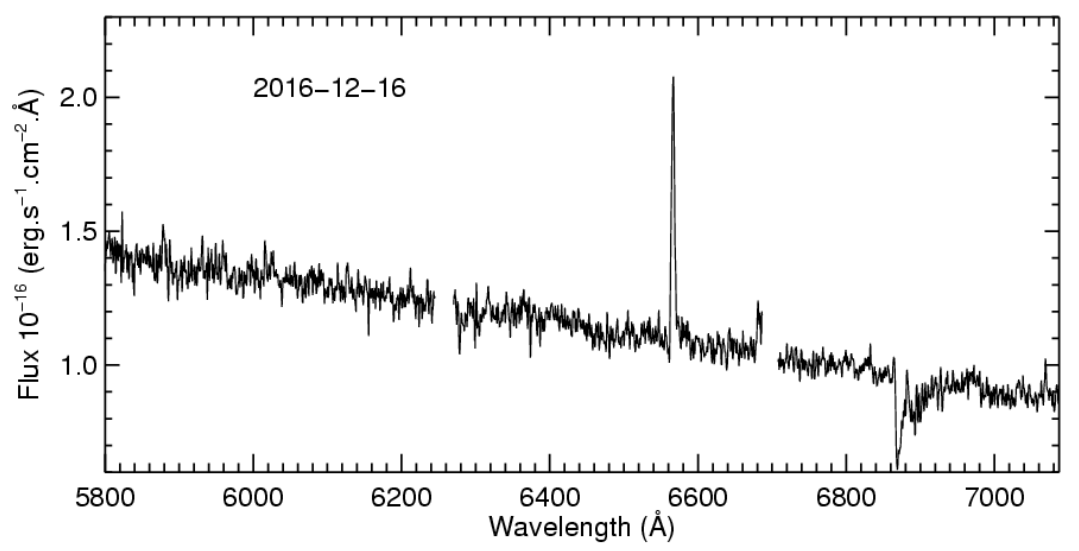

Figure 3: A red SALT spectrum (PG1800 grating) of ASASSN-16oh. The dominant emission line is $\mathrm{H} \alpha$.

ASASSN-16oh has also been monitored by OGLE, from prior to discovery and back to quiescence (Fig. 3). Several smaller amplitude outbursts are evident in the OGLE light curve, but what is most interesting is the symmetrical nature of the slow outburst and decline (which lasted $\sim 200 \mathrm{~d}$ ), like an inverted $\mathrm{V}$, which is quite different to the usual rapid rise and slow decline of SSSs. Our LCO photometry, obtained over a $\sim 2$ day time span, indicated a possible periodicity at $4.62 \mathrm{hr}$ (or one day aliases), which was also consistent with the HeII radial velocity modulations. However, with the rather inadeqaute sampling, these results are unreliable and a longer period (5.6 days) could equally well explain the results. This would also be more consistent with the energetics of the system, particularly the measured absolute magnitude, $M_{V}$, X-ray luminosity, $L_{X}$ and orbital peripd, $P$. A correlation between $M_{V}$ and $\log \Sigma\left(\Sigma=L_{X}{ }^{1 / 2} P^{2 / 3}\right)$ was shown to exist for LMXBs [12] and SSSs [13], and for ASASSN-16oh an orbital period of $5.6 \mathrm{~d}$ is more consistent with this correlation than an alternative shorter period.

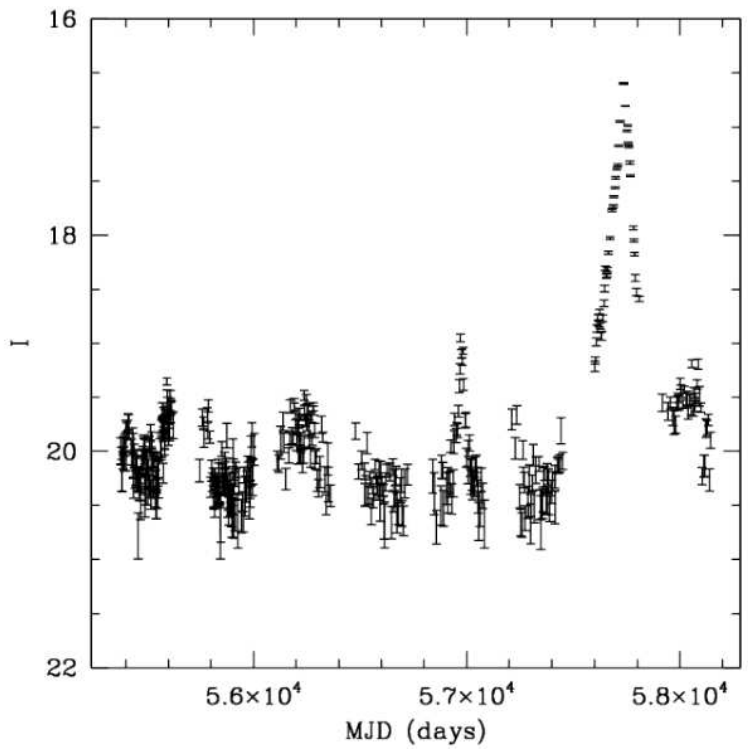

Figure 4: OGLE I-band light curve of ASASSN-16oh showing the large 2016 outburst plus evidence for smaller outbursts prior to this. 


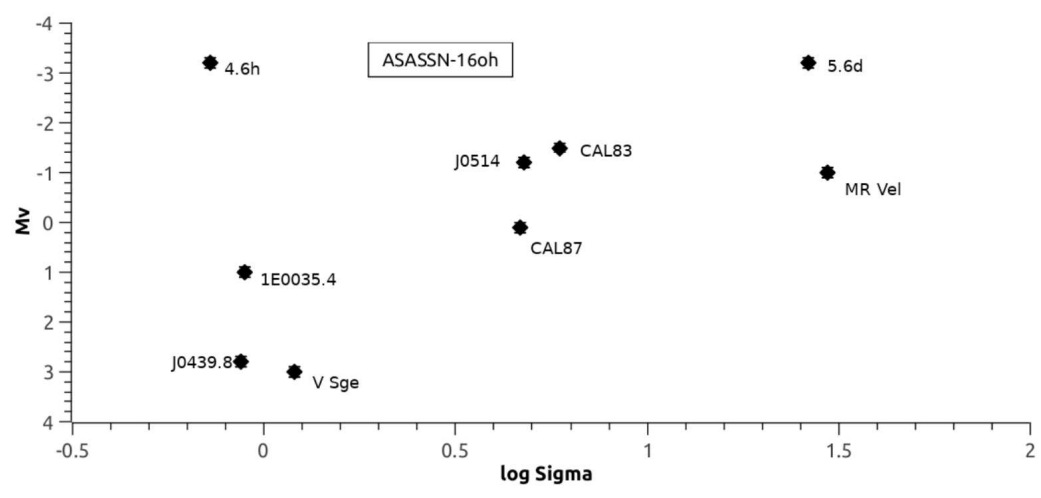

Figure 5: The absolute magnitude vs $\log \Sigma$ relationship [12], plotted for all known SSS (e.g. after [13], including for the newly identified ASASSN-16oh. The top two points correspond to the two proposed orbital periods values of $4.6 \mathrm{~h}$ (left) and the more likely period of $5.6 \mathrm{~d}$ (see text for details).

Work has continued on ASASSN-16oh, which has now declined to quiescence (V 21). It would appear that this is the most luminous SSS yet detected and furthermore that the nature of the outburst, with its slow symmetrical rise and decline, is not that expected for ignition of nuclear burning. The results have recently been interpeted, instead, as evidence for a hot spreading layer [14] on the WD following enhanced mass transfer, which is presented in our discovery paper on this source [15].

\subsection{Low Mass X-ray Binary transients}

\subsubsection{MAXI J1957+032}

This high-latitude transient was initially discovered by the MAXI instrument on the ISS in May 2015, but its X-ray outburst was very short-lived (undetectable within 5 days of the outburst). Three more, equally fast, outbursts occurred in Oct 2015, Jan and Sep 2016, and in the latter we were able to obtain low resolution SALT spectra of the optical counterpart (Figure 6). What makes this transient particularly interesting is the frequency of the outbursts and its rapid decline. Furthermore, a proposed identification with an $\sim F$ star was made in Oct 2015 using Nordic Optical Telescope (NOT) spectra, which was very unusual, as it was already recognized that there was no optical counterpart in quiescence on existing sky surveys, and so the optical brightness had increased by at least 4 magnitudes during the outburst, meaning that it must be an LMXB with a faint, low-mass donor. At such times, LMXB transient counterparts are dominated by their X-ray irradiated accretion discs, and so the proposed NOT identification was extremely odd.

The SALT spectrum immediately resolved this problem when it was found to be featureless, typical of X-ray luminous LMXBs. Reanalysis of the NOT data indicated that, as a result of the extremely rapid fading of J1957+032, the original optical counterpart was misidentified. Furthermore, we exploited the MAXI and Swift X-ray light-curves of the 4 very rapid outbursts to propose that this source is actually an AMXP, an Accreting Millisecond X-ray Pulsar. Their light-curves in decline exhibit a steepening below a critical luminosity level, as a result of the magnetospheric barrier that is presented by the rapidly rotating pulsar. If confirmed, this is likely to become an important member of this class, as its frequent outbursts combine 
with its high galactic latitude to make it observable over a much wider range of wavelengths than other AMXPs. Results of this investigation have appeared in [16] and [17].

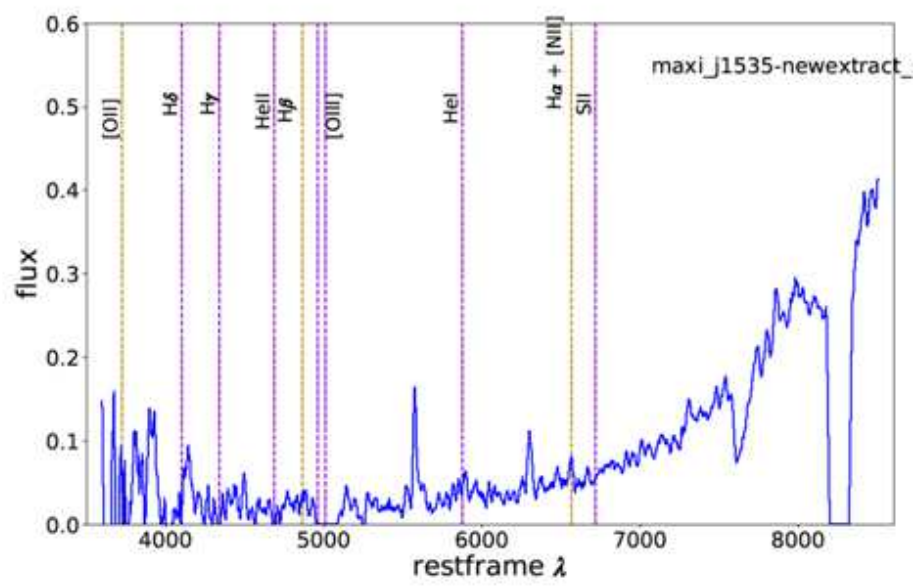

Figure 6: SALT spectrum of the optical counterpart of MAXI J1957+032 [17]. Apart from telluric lines, there are no other features seen in the spectrum.

\subsubsection{Swift J1357-0933}

This high-latitude, faint X-ray transient has one of the shortest orbital periods $(2.8 \mathrm{~h})$, but stood out for its astonishing variable QPO (of order $\sim 10^{2} \mathrm{~s}$ ) that was discovered in the 2011 outburst [18]. The source again went into outburst in April 2017, and we have successfully obtained SALT spectra showing broad double-peaked HeII emission, as well as an indication of Bowen emission. Furthermore fast photometry revealed QPOs, in the form of intensity dips (see Figure 7), evolving to lower frequencies over a period of $\sim 1$ month (see power spectrum in Figure 8), as was also seen in the 2011 outbursat [18]. The first SALT observation (28 Apr 2017) was simultaneous with an X-ray observation with NuSTAR, allowing for an autocorrelation analysis to be done to detect any time lags. Interestingly there was little correlation between X-rays and optical, suggesting the sources of these emissions are not necessarily connected. A SALT spectropolarimetric observation was also undertaken, to test the hypothesis that an accretion torus (as hypothesised in [18]) would give rise to polarized emission, which was not detected.

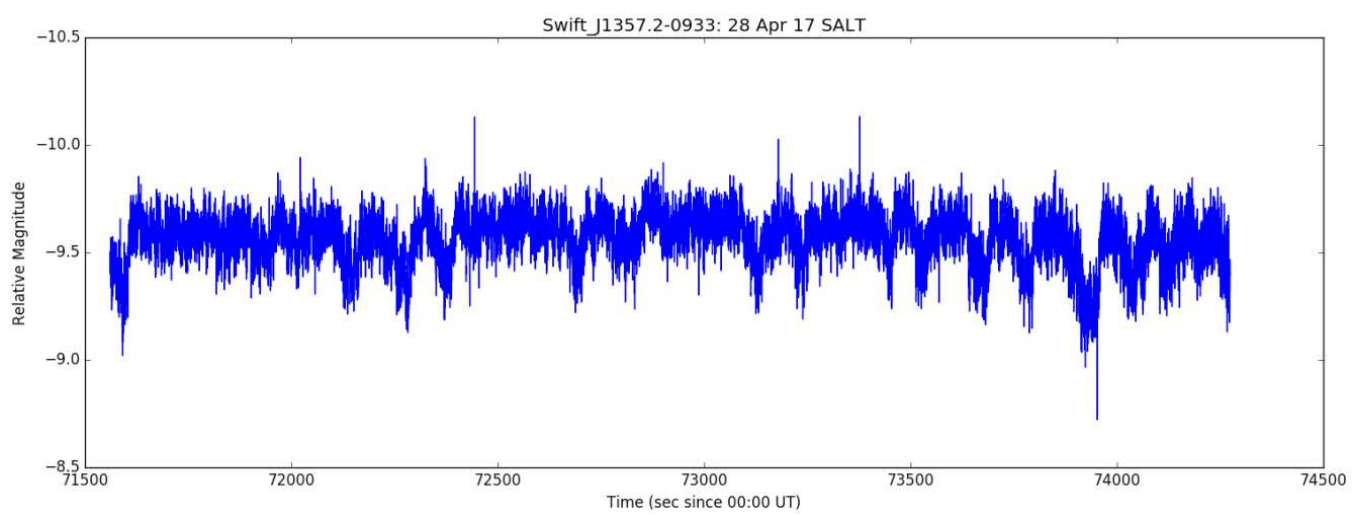

Figure 7: SALT slot-mode high speed photometry (0.1 s exposures) of Swift J1357-0933, taken on 28 April 2017. Quasi-periodic dips are seen from time to time on a $100 \mathrm{~s}$ timescale. 


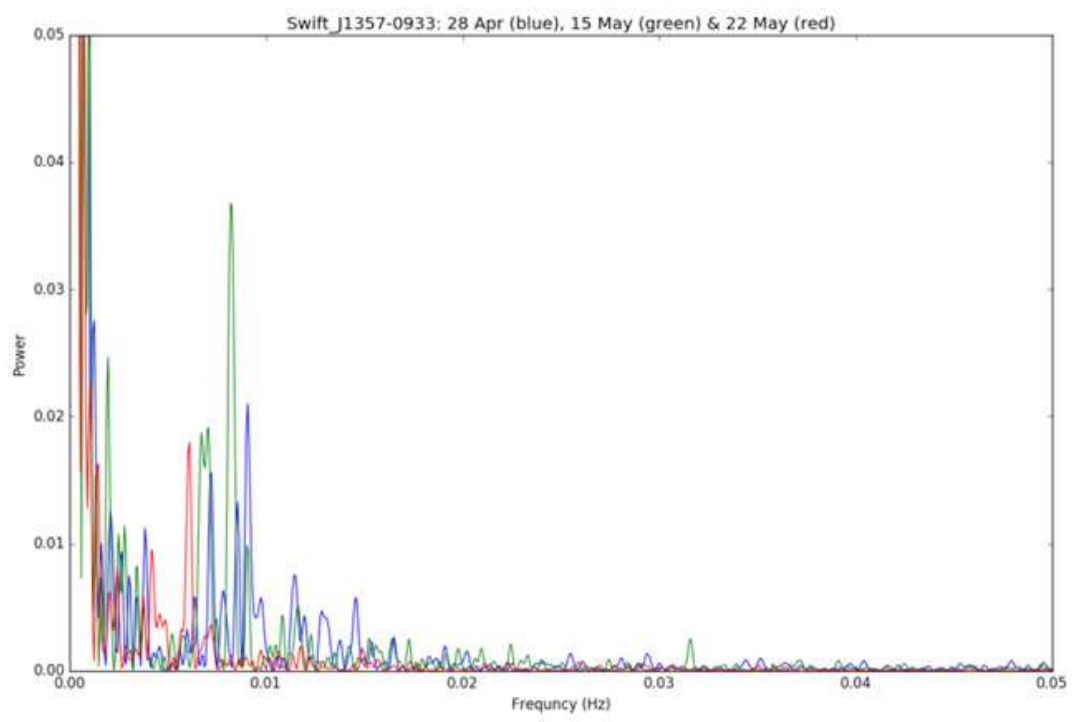

Figure 8: A Lomb-Scargle power spectrum of SALT RSS high-speed slot mode photometry of Swift J1357-0933, taken over a period of $\sim 1$ month, namely on 28 April (blue), 15 May (green) and 22 May 2017 (red). The dip QPO is seen to evolve to lower frequencies over this time interval.

\subsection{The Super-Eddington Outburst of SMC X-3}

Observations of the Be/X-ray binary pulsar SMC X-3 were obtained during the recent (2016) super-Eddington outburst. This was one of the brightest and longest outbursts ever observed from a Be/X-ray binary and was heavily monitored by the Swift satellite to observe the X-ray behaviour and measure the system parameters for the first time.

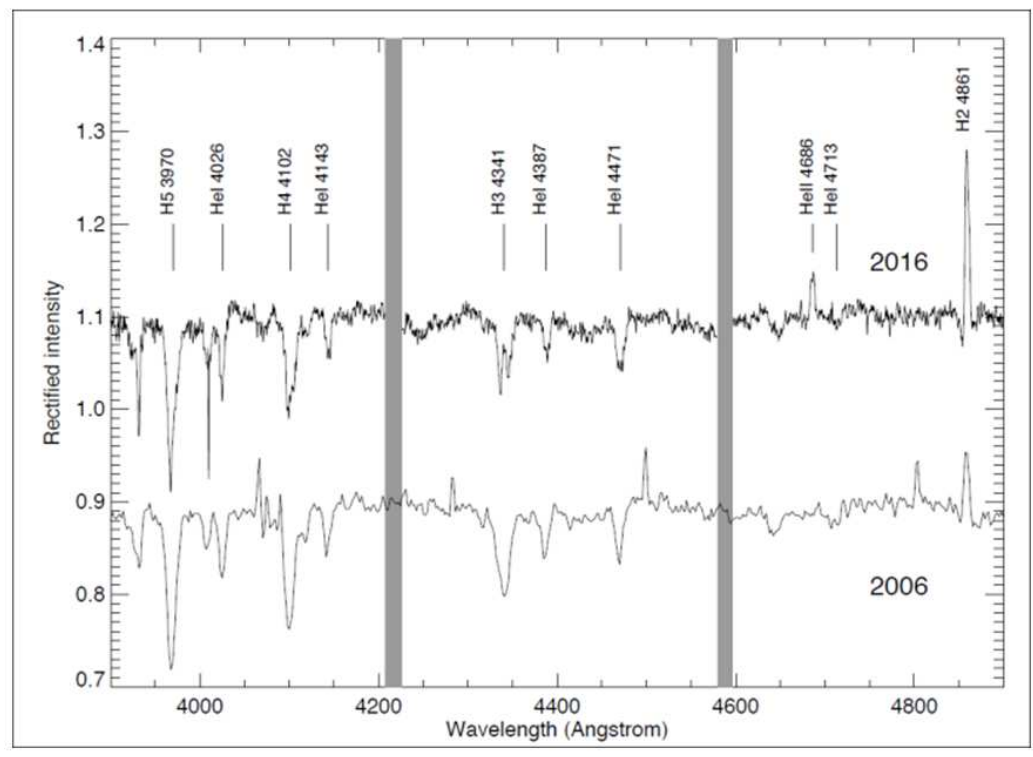

Figure 9: Spectral evolution of SMC X-3, showing the ESO 3.6m (2006) and SALT (2016) spectra, the latter taken during the current super-Eddington outburst [19]. 
In comparison to a previous quiescent spectrum, taken with the ESO 3.6-m telescope, the SALT RSS blue spectrum (Figure 9) revealed a strong enhancement of the $\mathrm{H} \beta$ emission and an infilling of the $\mathrm{H} \gamma$ absorption line during the outburst. This was interpreted as evidence that the circumstellar disc around the Be star had grown, causing the onset of the giant X-ray outburst from increased accretion of disc material onto the neutron star. The RSS spectrum also showed He II $4686 \AA$ in emission, which was not present in archival spectra. This provided good evidence that a variable and 'clumpy' accretion disc had formed around the neutron star. This explains why the X-ray spin period observed by Swift was highly variable, beyond the level expected from spin-up at a constant accretion rate. These results were published in 2017 [19].

\section{Conclusions}

The SALT Large Science Programme on transients is making important contributions to the study of X-ray transients across a range of classes, including HXMBs, LMXBs and SSSs. SALT's ability to react quickly to outburst alerts and employ a variety of observational techniques, coupled with its ability to monitor such transients during their outburst and decline, shows one of the competitive advantages of the SALT.

We have observed $12 \mathrm{X}$-ray transients to date, obtaining some good result on 9 (3 were too faint to be useful), which to date have resulted in 2 published paper, 2 papers in press and several in preparation. Several of the results of these investigations are described in this paper.

The future of transient research looks promising and SALT together with other telescopes at the SAAO, will play an ever increasing and important role.

\section{Acknowledgments}

The SALT transients programme benefits from a large number of collaborators who collectively have made the programme extremely successful. We wish to acknowledge the excellent support of the SALT operations staff and in particular to Christian Hettlage who has assisted on numerous occasions with observing block submission and software issues.

The results presented here were taken under the SALT Large Science Programme, 2016-2LSP-001, which is supported by South Africa, the UK SALT Consortium, the Indian InterUniversity Centre for Astronomy and Astrophysics, the University of Wisconsin-Madison and Poland, the latter funded through grant no. MNiSW DIR/WK/2016/07. DB acknowledges the support of the National Research Foundation.

\section{References}

[1] D. A. H. Buckley et al., 2006, Proc. SPIE 6269, 62690

[2] J. Casares, 2007, IAU Symp 238, 3

[3] M. Bachetti M. et al., 2014, Nature, 514, 202

[4] D. Steeghs \& J. Casares, 2002, Ap. J., 568, 273

[5] R. Cornelisse et al., 2008, AIPC, 1010, 148. 
[6] P. A. Charles \& M. Coe, 2006, in Compact Stellar X-ray Sources, Cambridge Astroph. Series No.39, 215

[7] R. Fender \& T. Belloni, 2012, Sci, 337, 540

[8] L. Townsend, et al $2017 \mathrm{MN} 471,3878$

[9] K. L. Li, et al., 2012, ApJ, 761, 99

[10] B. J. Shappee, et al., 2014, ApJ, 788, 48

[11] S.W. Jha, S.W., et al., 2016, Astronomer's Telegram, 9859

[12] J. Van Paradijs \& J. E. McClintock, 1994, A\&A 290, 133.

[13] A. van Teeseling, et al., 1997, A\&A 323, L41.

[14] A. L. Piro \& L. Bildsten, 2004, ApJ, 610:977-990

[15] T. Maccarone, et al., 2018, Nat. Ast, in press.

[16] D. A. H. Buckley, et al., 2016, Astronomer's Telegram, 9649

[17] D. Mata Sánchez, et al., 2017 MNRAS 468, 564

[18] J. M. Corral-Santana, et al., 2013, Science, 339, 1048 